\section{Sobrevida en carcinoma espinocelular de labio}

\author{
TANIA CABELLO B. ${ }^{a}$, NICOLÁS SAZO B. ${ }^{a}$, \\ APOLO SALGADO F. ${ }^{1}$, BENJAMÍN MARTÍNEZ R. ${ }^{2}$
}

\section{Squamous cell carcinoma of the lip survival rate}

Background: Squamous Cell Carcinoma (SCC) of the lip represents about $20 \%$ of all oral carcinomas. It is more common in men, peaking during their sixth and seventh decade, and is more prevalent in the lower lip. Aim: To determine the five years survival rate of Chilean patients with lip SCC. Material and Methods: The registry of the Chilean National Cancer Institute was reviewed to retrieve the clinical records of patients with lip SCC. Information about age, sex, alcohol consumption, smoking, degree of pathological differentiation, lip location, type of treatment and metastases was recorded. Survival was determined with death certificates from the National Identification Service. Results: Seventy four patients were identified. Their 5-year survival was 73\%. Conclusions: The survival rate of this group of patients is lower than that of patients from Unites States who have an $89.5 \%$ survival, according to the Surveillance, Epidemiology, and End Results Program of the North American National Cancer Institute.

(Rev Med Chile 2015; 143: 847-855)

Key words: Carcinoma, Squamous Cell; Lip neoplasms; Survival analysis; Risk factors.

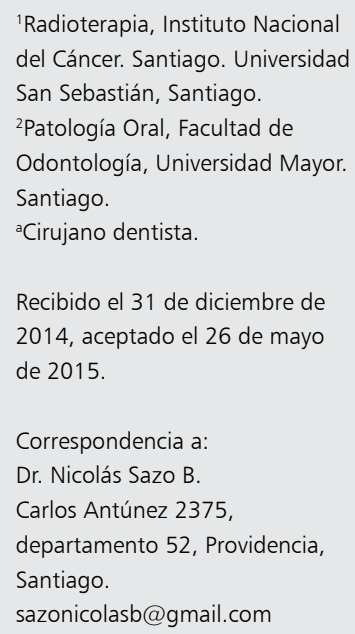

$\mathrm{E}$ 1 cáncer a nivel oral representa aproximadamente 3\% de todos los tumores malignos $\checkmark$ posibles de desarrollar convirtiéndose en el sexto cáncer más común en el mundo ${ }^{6,18}$. En Chile, el cáncer oral y de labio según el proyecto GLOBOCAN $^{19}$ tuvo una incidencia de 1,2 por cada 100.000 habitantes. En la Tabla 1 se detalla la incidencia y mortalidad del cáncer oral y labial en Chile.

El carcinoma espinocelular (CEC) es el tumor maligno más frecuente en la cavidad oral ${ }^{14}$ con un porcentaje de $94 \%{ }^{8}$, siendo su localización más común la lengua ${ }^{18}$. El riesgo de presentar cáncer oral aumenta con la edad y se manifiesta mayormente en personas sobre 50 años, siendo más afectado el género masculino ${ }^{18}$.

El carcinoma a nivel oral es una enfermedad multifactorial cuya aparición tiene estrecha relación con hábitos de los pacientes, como son el consumo de tabaco y alcohol ${ }^{8,12}$. También son factores de riesgo el virus papiloma ${ }^{6,12,18}$, sífilis terciaria ${ }^{8}$, deficiencias nutricionales (insuficiencia de hierro y vitamina $A)^{8,12}$, candidiasis, liquen plano, factores hereditarios, higiene oral deficiente $\mathrm{e}$ incluso desigualdades sociales ${ }^{12}$, entre otros.

El tratamiento para un paciente con cáncer es variado, contando con cirugía, radiación o quimioterapia, solas o combinadas. La elección de un determinado tratamiento varía según factores asociados al tumor, como su localización, proximidad a tejido óseo, profundidad de invasión y etapa en la que se encuentre (en relación al tamaño, invasión de nódulos linfáticos y metástasis). Otras consideraciones son edad del paciente, co-morbilidades que éste presente y complicaciones propias del tratamiento ${ }^{10}$.

El CEC de labio representa cerca de $20 \%$ de los carcinomas orales ${ }^{16}$, siendo más común en hom- 
Tabla 1.

\begin{tabular}{|ccccc|}
\hline Región & $\begin{array}{c}\text { Incidencia en } \\
\text { hombres }\end{array}$ & $\begin{array}{c}\text { Incidencia en } \\
\text { mujeres }\end{array}$ & $\begin{array}{c}\text { Mortalidad en } \\
\text { hombres }\end{array}$ & $\begin{array}{c}\text { Mortalidad en } \\
\text { mujeres }\end{array}$ \\
\hline Labio y cavidad oral & 1,6 & 0,8 & 0,8 & 0,3 \\
\hline
\end{tabular}

bres y con un peak de ocurrencia entre la sexta y séptima década, además, es más prevalente en el labio inferior que en el superior ${ }^{8}$.

A nivel del labio, se distinguen el CEC del borde bermellón, mucosa labial y comisura, existiendo en cada uno distintos factores para su aparición, siendo la ubicación más común el borde de bermellón. Éste por lo general se presenta en personas con piel clara que permanecen muchas horas al día expuestos a radiación $\mathrm{UV}^{8}$ y con historia de daño causado por el sol a temprana edad $^{8}$, considerándose el factor de mayor riesgo la exposición crónica al sol como ocurre en trabajos en sectores rurales o al aire libre ${ }^{7}$. Esta exposición puede desarrollar queilitis actínica a nivel del labio inferior, lesión que es considerada potencialmente maligna y puede transformarse en un carcinoma espinocelular ${ }^{15}$. Otros factores de riesgo son fumar tabaco, presencia de virus (EBV, CMV, HSV, $\mathrm{HPV}$ ), factores genéticos, inmunosupresión y estado socioeconómico ${ }^{7}$.

Las lesiones se presentan en labio inferior entre comisura y línea media con un aspecto de costra, no dolorosa, que puede o no estar ulcerada y con bordes indurados que suele ser de menos de un $\mathrm{cm}$ en su diámetro mayor cuando es descubierta ${ }^{8}$.

El objetivo de este trabajo fue analizar la sobrevida a los cinco años de una muestra de pacientes diagnosticados con CEC de labio, comparando estas cifras con los valores de otros estudios. Además, se analizará cómo afectan a la sobrevida distintos factores.

\section{Materiales y Métodos}

Se revisaron los libros del Comité del Instituto Nacional del Cáncer de Chile (INC), de donde se extrajo el número de ficha de los pacientes con diagnóstico de cáncer de labio para luego confirmar el diagnóstico en las fichas. El criterio de inclusión para la muestra del estudio fue: pacientes diagnosticados con carcinoma espinocelular de labio mediante biopsia. Se excluyeron aquellos pacientes diagnosticados con carcinoma espinocelular en otras localizaciones y otros tipos histopatológicos de cáncer labial.

Los datos obtenidos de las fichas se tabularon en una planilla Excel $^{\circledR}$, considerando nombre completo, RUT, edad (años), género ( $M$ ó F), alcohol (sí/no), tabaco (sí/no), grado de diferenciación histopatológica (bien, moderado y poco diferenciado), localización, tipo de tratamiento (cirugía y quimioterapia/cirugía y radioterapia/ cirugía, radioterapia y quimioterapia, otros) y presencia o no de metástasis. No se consideró TNM debido a la ausencia de esta información en la mayoría de las fichas. Se obtuvo un total de 74 pacientes diagnosticados entre febrero de $1996 \mathrm{y}$ abril de 2014. Se determinó qué pacientes habían fallecido, mediante certificado de defunción con causa de muerte, obtenido del Registro Civil de Chile. Se analizó la relación existente entre porcentaje de sobrevida y presencia de los factores asociados más relevantes.

Para el análisis estadístico de sobrevida se utilizó el método Kaplan-Meier en el programa STATA V.13.1. También se utilizó el test rango de logaritmo para comparar las curvas de sobrevida y determinar si existían diferencias significativas; se consideró que las había si p $<0,05$.

\section{Resultados}

Del total de 74 pacientes y a través del certificado de defunción del Registro Civil, se determinó la causa de muerte y el número de pacientes fallecidos. Aquellos pacientes en que el sistema no generó certificados se consideraron vivos.

Hubo 46 pacientes fallecidos (62\%), de los cuales 17 (37\%) tuvieron causa de muerte el cáncer de labio, mientras que los otros 29 (63\%) murieron por otra causa; 28 (38\%) de 74 permanecían vivos. La sobrevida media de los pacientes fallecidos fue de 2,7 años (rango 0,1 a 12,9 años). La sobrevida media de los pacientes que permanecían vivos fue 5,6 años (DS: 4,1) (rango 0,3 a 18,1 años). 

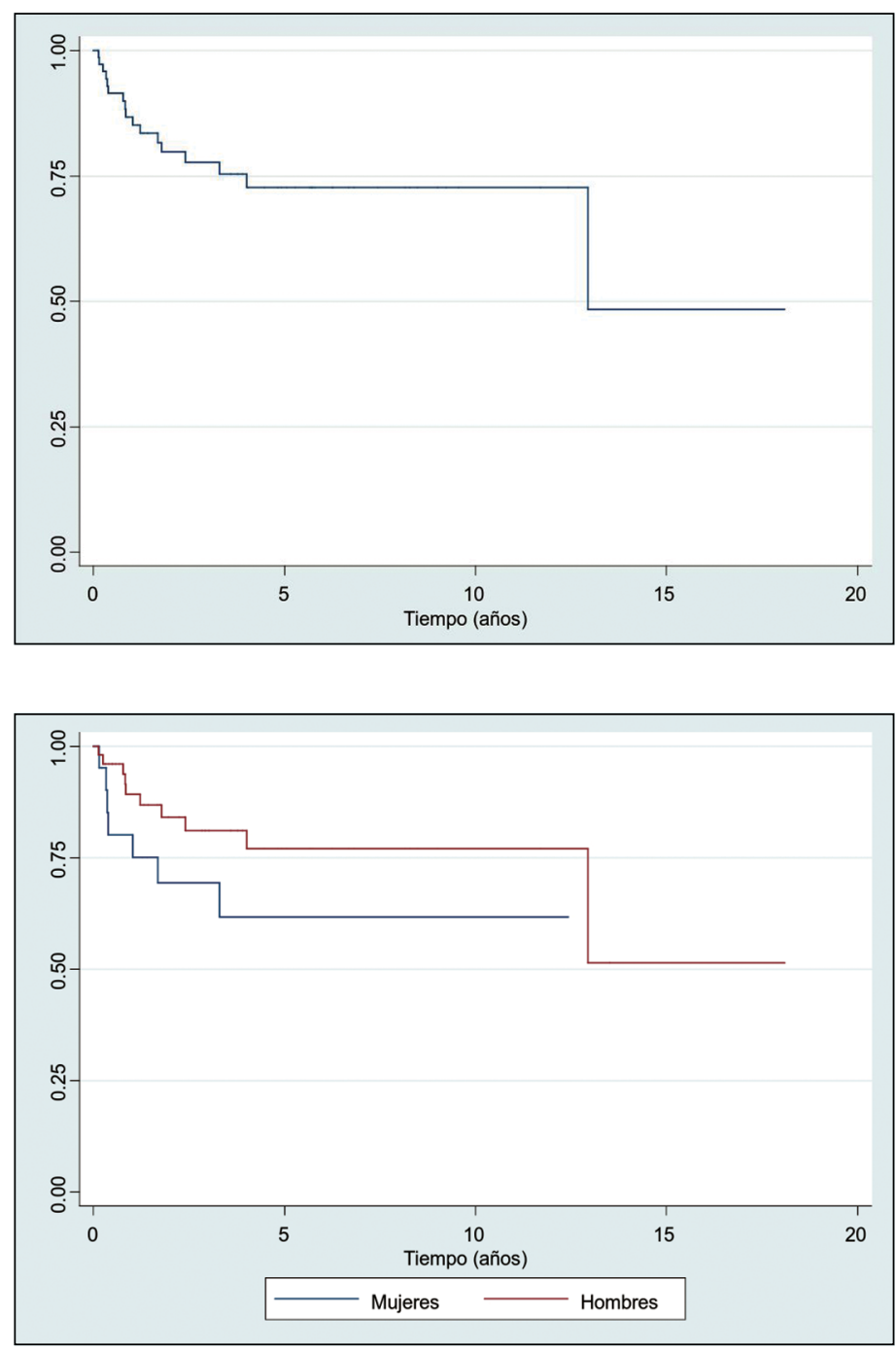

Figura 1. Curva de sobrevida Kaplan-Meier en pacientes del Instituto Nacional del Cáncer diagnosticados con Carcinoma Espinocelular de Labio $(n=74)(1996-2014)$. En la presente curva de sobrevida de Kaplan-Meier de todos los pacientes diagnosticados con CEC de labio del Instituto Nacional del Cáncer, se observó una sobrevida a los cinco años de $73 \%$ y que se mantuvo más allá de los diez años.
Figura 2. Curva de sobrevida Kaplan-Meier según género, en pacientes del Instituto Nacional del Cáncer diagnosticados con Carcinoma Espinocelular de Labio (1996-2014). En esta curva de sobrevida Kaplan-Meier según género, se contabilizaron mujeres $(n=21)$ en azul y hombres $(n=53)$ en rojo. A pesar de que existe mayor cantidad de hombres que mujeres diagnosticados con CEC labial, no existieron diferencias significativas con respecto a la sobrevida (Log Rank Test, $\mathrm{p}=0,1615$ ).
La sobrevida del cáncer labial en Chile fue de $73 \%$ a los cinco años (Figura 1). No se encontraron diferencias significativas (Log Rank Test $\mathrm{p}=0,1615)$ al relacionar sobrevida y género (Figura 2). El promedio de edad fue de 72 años (DS: 11,50). No hubo diferencias significativas entre grupos de edad y sobrevida del carcinoma labial (Log Rank Test $\mathrm{p}=0,70$ ) (Figura 3). Los pacientes no consumidores de alcohol tuvieron $70 \%$ de sobrevida a los cinco años, mientras que consumidores presentaron sobrevida de cerca de 75\% (Figura 4), sin embargo, no existían diferencias significativas (Log Rank Test $\mathrm{p}=0,7746)$. Los pacientes consumidores de tabaco presentaron mayor sobrevida a los cinco años que los no con- sumidores (Figura 5), sin haber diferencias significativas entre ellos ( Log Rank Test $\mathrm{p}=0,5971)$. Con respecto a localización de la lesión y sobrevida existieron diferencias significativas (Log Rank Test $\mathrm{p}=0,0316$ ) donde el bermellón del labio inferior presentó sobrevida a los cinco años de 74\% (Figura 6). El grado de diferenciación histopatológica de la lesión tuvo relación con sobrevida de los pacientes, encontrándose diferencias significativas ( $\log$ Rank Test $\mathrm{p}=0,0175$ ). Aquellos con carcinoma pobremente diferenciado tuvieron peor sobrevida, en comparación con el bien diferenciado (Figura 7). La presencia de metástasis fue estadísticamente significativa ( $\log$ Rank Test $\mathrm{p}=0,0105)$ (Figura $8)$. Los tratamientos realizados fueron cirugía 

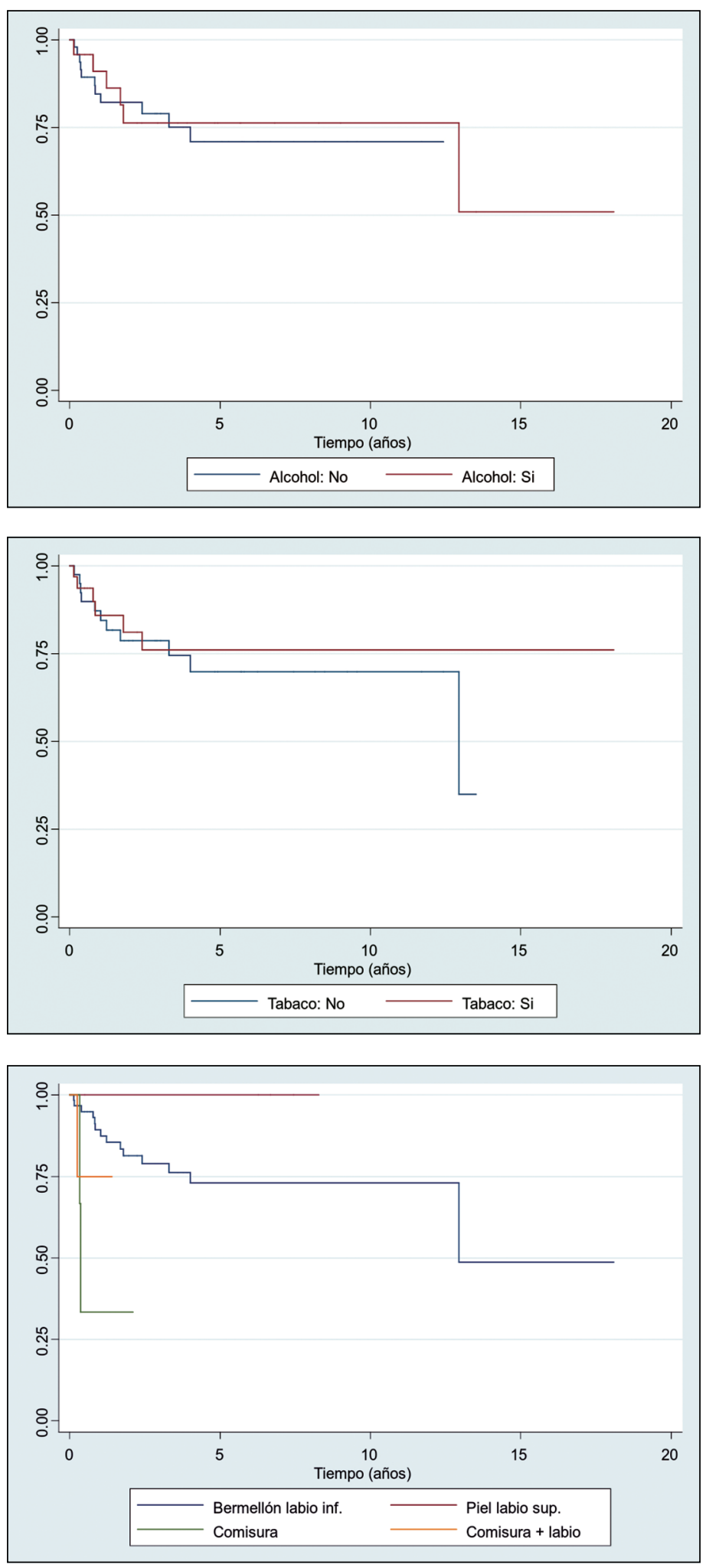

Figura 3. Curva de sobrevida Kaplan-Meier según consumo de alcohol, en pacientes del Instituto Nacional del Cáncer diagnosticados con Carcinoma Espinocelular de Labio (19962014). En esta curva de sobrevida Kaplan-Meier según consumo de alcohol, se observa una curva azul que representa a los 50 pacientes que relataron ser no bebedores y la curva roja representa a los 24 pacientes que sí eran bebedores. No existieron diferencias significativas entre los grupos y la sobrevida (Log Rank Test, $p=0,7746)$.

Figura 4. Curva de sobrevida Kaplan-Meier según consumo de tabaco, en pacientes del Instituto Nacional del Cáncer diagnosticados con Carcinoma Espinocelular de Labio (19962014). En esta curva de sobrevida Kaplan-Meier según consumo de tabaco, al igual que en la figura anterior, la curva azul representa a 42 pacientes que no son consumidores de tabaco y la curva roja representa a 32 pacientes que eran consumidores de tabaco. No existieron diferencias significativas entre los grupos y la sobrevida (Log Rank Test, $\mathrm{p}=0,5971$ ).

Figura 5. Curva de sobrevida Kaplan-Meier según distintas ubicaciones labiales, en pacientes del Instituto Nacional del Cáncer diagnosticados con Carcinoma Espinocelular de Labio (1996-2014). En esta curva de sobrevida según distintas ubicaciones labiales, se observa una curva azul que representa a los pacientes que tenían el CEC en bermellón del labio inferior $(n=60)$, donde a los cincos años se observó $73 \%$ de sobrevida; en morado, la curva que representa a los que tenían el CEC en piel del labio superior $(n=5)$, quienes tuvieron una sobrevida de $100 \%$ a los cinco años y posteriormente murieron por otras causas; en verde, los pacientes que tenían el CEC en la comisura $(n=4)$ y por último, en amarillo aquellos que lo tenían en comisura más el labio $(n=5)$. Existieron diferencias significativas entre la ubicación del CEC labial y la sobrevida (Log Rank Test, $\mathrm{p}=0,0316$ ). 


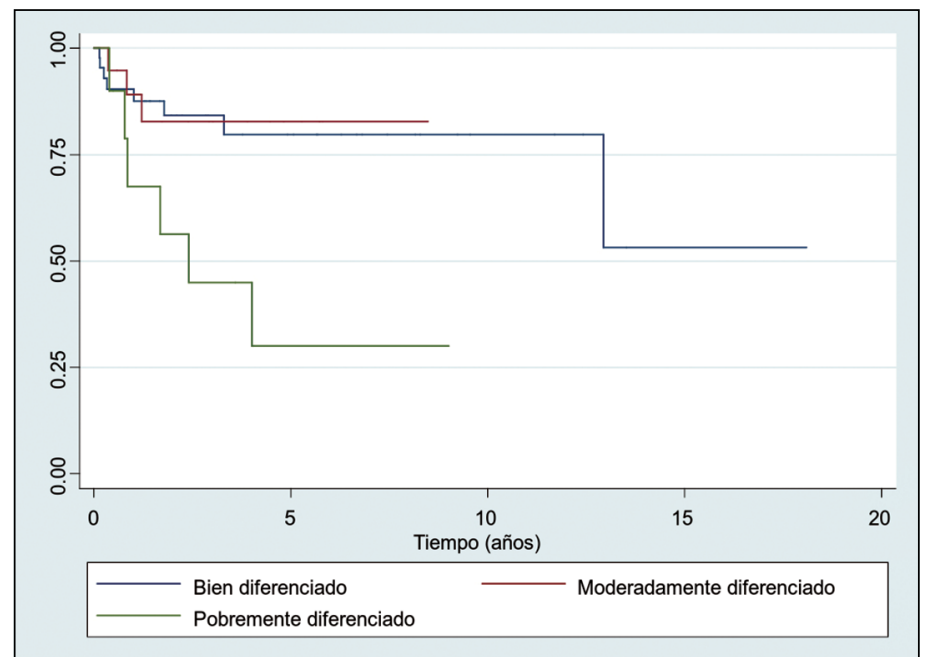

Figura 6. Curva de sobrevida Kaplan-Meier según grado de diferenciación de la lesión, en pacientes del Instituto Nacional del Cáncer diagnosticados con Carcinoma Espinocelular de Labio (1996-2014). En esta curva de sobrevida según grado de diferenciación de la lesión, la curva azul representa a los pacientes que presentaron el CEC bien diferenciado $(n=43)$, que presentaron una sobrevida de $80 \%$ a los cinco años; la curva en rojo representa a los pacientes que presentaron un CEC moderadamente diferenciado $(n=19)$ y por último en verde la curva que representa el CEC pobremente diferenciado $(n=$ 11), donde se observa una menor sobrevida a los cinco años llegando al 30\%. Existieron diferencias significativas entre el grado de diferenciación de la lesión y la sobrevida del CEC labial (Log Rank Test, p =0,0175). Sólo un caso no fue registrado.

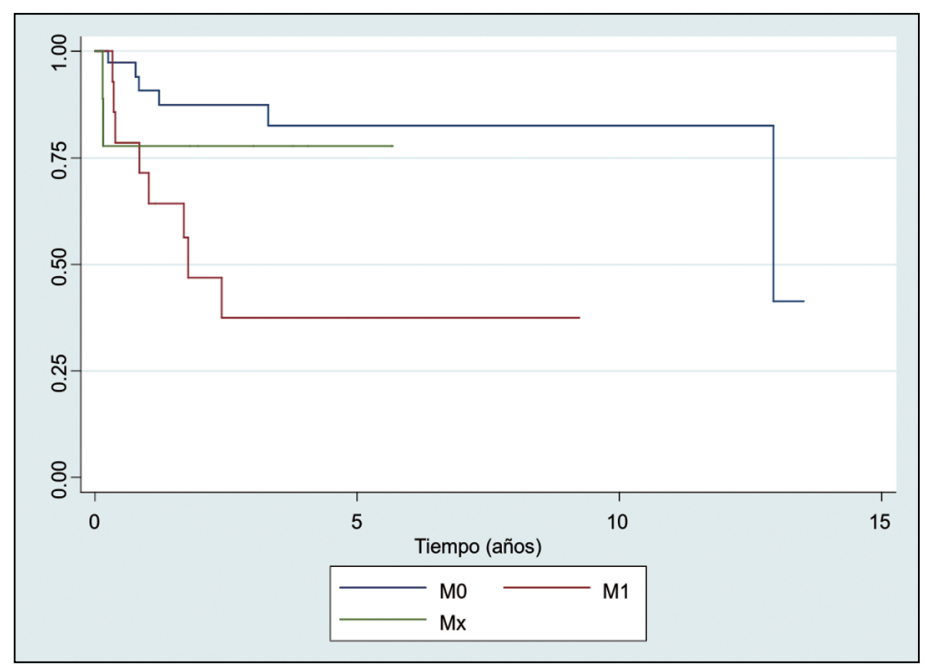

Figura 7. Curva de sobrevida Kaplan-Meier según metástasis, en pacientes del Instituto Nacional del Cáncer diagnosticados con Carcinoma Espinocelular de Labio (1996-2014), (M1: metástasis a distancia, M0: ausencia de metástasis a distancia, Mx: no se puede establecer metástasis a distancia). En esta curva de sobrevida Kaplan-Meier según metástasis, la curva azul representa a aquellos pacientes que no tuvieron metástasis a distancia, denominado M0 ( $n=39$ ), estos presentan la mayor sobrevida (80\%) de los tres grupos a los cinco años; la curva roja representa a los pacientes que tuvieron metástasis a distancia, denominado M1 ( $n=14)$, estos presentaron una sobrevida de sólo 37\%. La curva verde representa a aquellos pacientes en los cuales no se pudo establecer metástasis a distancia, denominados como $\mathrm{Mx}(\mathrm{n}=9)$. Existieron diferencias significativas entre la presencia de metástasis y la sobrevida del CEC labial (Log Rank Test, $\mathrm{p}=0,0105$ ). Hubo 12 casos que no fueron registrados. M0: no existe metástasis a distancia, M1: presencia de metástasis a distancia, Mx: no es posible evaluar una metástasis a distancia. 


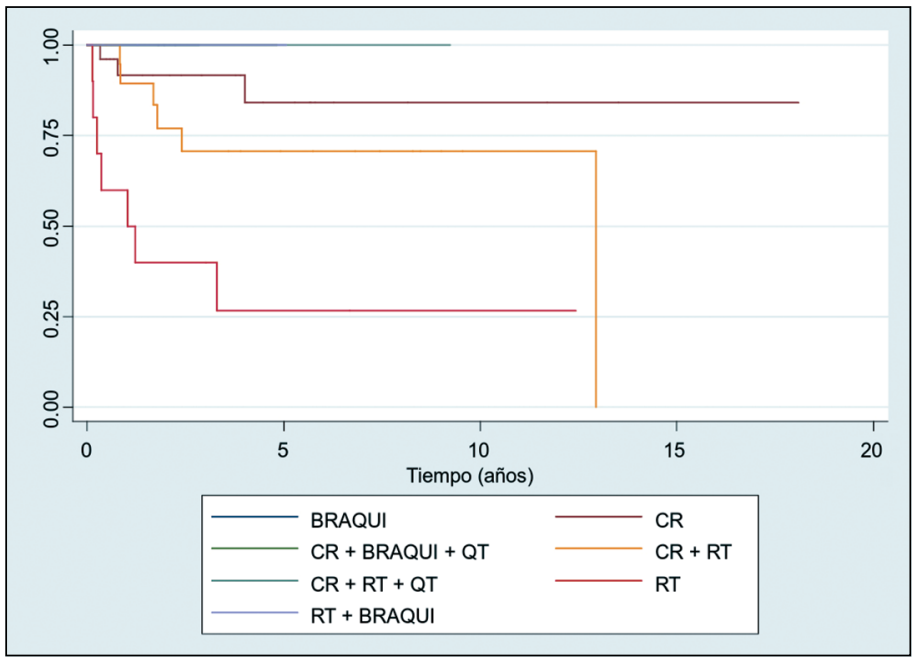

Figura 8. Curva de sobrevida Kaplan-Meier según tipo de tratamiento, en pacientes del Instituto Nacional del Cáncer diagnosticados con Carcinoma Espinocelular de labio (1996-2014). En esta curva de sobrevida Kaplan-Meier según tipo de tratamiento, la curva café representa a la Cirugía Resectiva $(C R)(n=27)$ quienes tuvieron una sobrevida de $80 \%$ a los cincos años; éste fue el tratamiento más realizado. La curva en amarillo representa la Cirugía Resectiva más Radioterapia $(R T)(n=22)$, la curva roja representa a la Radioterapia por sí sola $(n=10)$. En azul a aquellos pacientes que se les hizo Braquiterapia $(B Q T)(n=4)$; en verde aquel paciente que recibió $C R+B Q T+$ Quimioterapia $(Q T)(n=1)$, en celeste aquel paciente que recibió $C R+R T+Q T(n=1)$ y por último en morado representa a los pacientes que recibieron $\mathrm{RT}+\mathrm{BQT}(\mathrm{n}=3)$. En este caso sí existieron diferencias significativas entre el tipo de tratamiento y la sobrevida de los pacientes con CEC labial (Log Rank Test, p =0,0013). Hubo 6 casos que no fueron registrados. BRAQUI: Braquiterapia, CR: Cirugía Resectiva, QT: Quimioterapia, RT: Radioterapia.

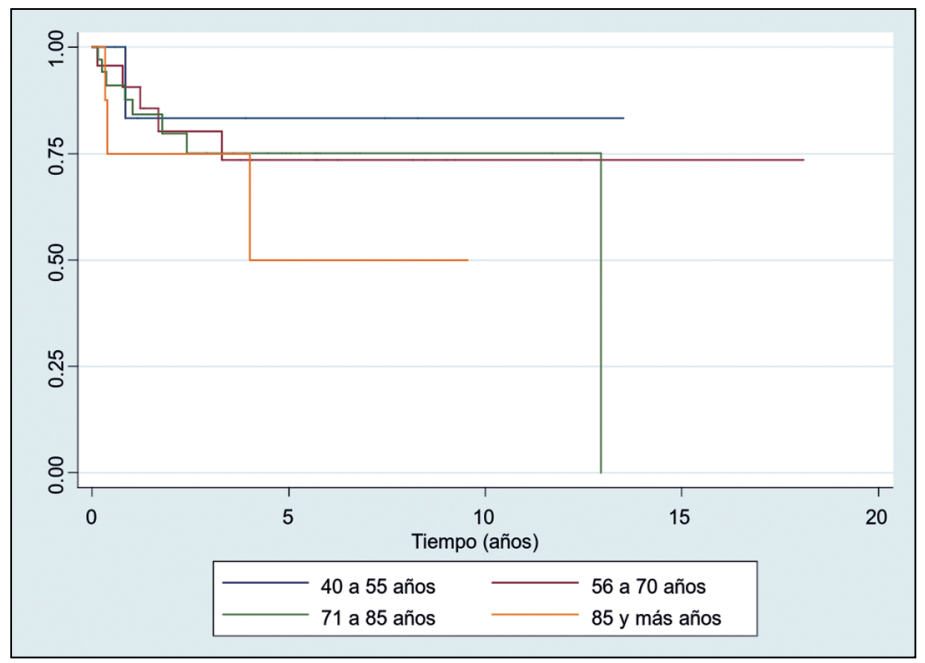

Figura 9. Curva de sobrevida Kaplan-Meier por grupos de edad, en pacientes del Instituto Nacional del Cáncer diagnosticados con Carcinoma Espinocelular de Labio (1996-2014). En esta curva de sobrevida Kaplan-Meier por grupos de edad, se representa en azul el grupo etario entre 40-55 años en el que hubo 8 pacientes, en violeta el grupo etario entre 56-70 años en el que hubo 23 pacientes, en verde el grupo etario entre 71-85 años en el que existieron 35 pacientes y por último en amarillo el grupo etario entre 85 y más años en el que hubo 8 pacientes. No hubo diferencias significativas entre el grupo etario y la sobrevida de los pacientes con CEC labial (Log Rank Test, $\mathrm{p}=0,70$ ). 
resectiva (27 casos), cirugía resectiva más radioterapia (22 casos), radioterapia por sí sola (10 casos), braquiterapia (4 casos) radioterapia más braquiterapia (3 casos) y cirugía resectiva más quimioterapia más braquiterapia con un caso, como también lo fue para cirugía resectiva más quimioterapia más radioterapia. El tratamiento realizado a los pacientes tuvo significancia estadística respecto a la sobrevida (Log Rank Test $\mathrm{p}=0,0013$ ), siendo radioterapia la que presentó la sobrevida más baja, a los cinco años $26 \%$; la cirugía resectiva fue el tratamiento más frecuentemente realizado (27 casos, correspondiente a $40 \%$ del total de pacientes tratados) con una sobrevida de $80 \%$ a los cinco años (Figura 9).

\section{Discusión}

El promedio de edad en nuestros casos fue similar a lo descrito por Gutiérrez-Pascual et al. (2011) (71,5 años de edad). La mayoría de los pacientes son diagnosticados de este carcinoma por sobre la quinta década de edad, como indica Czerninski et al. (2010) donde concluye que el diagnóstico fue por sobre los 53 años de edad en 75,5\% de los pacientes.

Encontramos mayor prevalencia en hombres que en mujeres (2,5:1, respectivamente), esto puede deberse a los cosméticos utilizados principalmente por mujeres que ayudan a proteger los labios de la radiación solar ${ }^{3,7}$. Pogoda et al. (1996) concluyó que, las mujeres que utilizaban lápiz labial tenían 50\% menos riesgo de tener cáncer de labio. Sin embargo, es necesario mencionar que nuestra proporción difiere a la expuesta por otros autores que indican que la proporción hombre-mujer corresponde a $6: 1^{1,13,15,21}$ lo que podría deberse a que teníamos menor cantidad de pacientes y también por el aumento de casos de cáncer de labio en mujeres. Esto último lo explica Moore et al. (1999) que concluye que en diferentes lugares del mundo, principalmente en Australia, existe un aumento en la incidencia de carcinoma labial en mujeres, así como también lo postula Yako-Suketomo y Marugame (2008).

Acerca del tabaco, se observó que la mayoría de los pacientes relataron no ser fumadores $(56,8 \%)$, a diferencia del estudio de Gutiérrez-Pascual et al. (2011) donde 72,3\% de los pacientes con CEC de labio relató ser fumador o haberlo sido en los últimos 20 años.
Un importante factor de riesgo para el CEC de labio es la radiación ultravioleta causando mutaciones en el $\mathrm{ADN}^{3}$. El desarrollo del tumor es el resultado de intentos fallidos de reparar estas mutaciones. La exposición al sol, por lo tanto, se considera un importante factor de riesgo para el desarrollo de estas lesiones de cáncer. Para Warnakulasuriya (2009), la exposición excesiva a luz ultravioleta se considera como factor de riesgo importante sólo para el cáncer de labio. La exposición crónica al sol es comúnmente citada como el mayor factor de riesgo en el desarrollo de cáncer de labio según Pukkala et al. (1994). Se considera que las personas que trabajan al aire libre presentan mayores tasas de cáncer labial, por los daños acumulativos en el tiempo ${ }^{2}$.

En nuestro estudio no se pudo evaluar esta variable debido a que esta información no estaba disponible en las fichas clínicas y porque es difícil registrar con exactitud esta variable ya que se cuenta sólo con medidas indirectas como ocupación o lugares de residencia. Sin embargo, consideramos que la radiación ultravioleta sí es el factor más importante en la aparición de lesiones de cáncer de labio, esto en base a la información mencionada por los autores. Si bien, el tabaco y alcohol son los factores de riesgo más importantes en el desarrollo de cáncer oral en general, en el caso particular del cáncer labial es importante señalar que el factor de riesgo más importante es la radiación ultravioleta aunque en ocasiones también se asocia el tabaco.

El cáncer labial se puede presentar en bermellón del labio inferior, labio superior, comisura y comprometiendo comisura más labio. En nuestro estudio, la ubicación de la lesión más prevalente fue el bermellón del labio inferior (81\%) al igual que Gutiérrez-Pascual et al. (2011) que observó que $91,1 \%$ de los pacientes presentaban la lesión en esta zona. Moore et al. (1999) señala que, a pesar de la dificultad de localizar la lesión primaria, la mayoría de las lesiones se ubican en el labio inferior. Vieira et al. (2012) por su parte indica que esta ubicación de la lesión es veinte veces más prevalente que en el labio superior. Neville (2009) señala que 90\% de las lesiones de carcinoma de labio se ubican en el bermellón del labio inferior; además, $70 \%$ de las personas que presentan el carcinoma trabajan al aire libre, lo que se asocia a queilitis actínica y una posible malignización de esta lesión. En la localización bermellón del labio inferior, se observó 74\% de 
sobrevida a los cinco años, lo que se puede interpretar como que, si bien es la zona más expuesta a la radiación solar, pareciera ser más favorable que las otras localizaciones.

Según el grado de diferenciación de la lesión, la mayoría de los pacientes presentó un carcinoma labial bien diferenciado (58,9\%), como también lo demuestra Gutiérrez-Pascual et al. (2011) donde $75 \%$ de las lesiones tenían esta característica histopatológica. De Visscher et al. (1998) señala que $93,5 \%$ de los tumores fueron bien y moderadamente diferenciados. En nuestro estudio sí existieron diferencias significativas con respecto a la sobrevida $(\mathrm{p}=0,0175)$, siendo el carcinoma labial pobremente diferenciado el que registró los fallecimientos más rápidos. Esto se debe a que mientras menos diferenciado sea el carcinoma, histopatológicamente presentará mayor pleomorfismo celular y nuclear, con poca o nula producción de queratina, numerosas mitosis típicas y atípicas; tendrá un crecimiento mucho más rápido y las posibilidades de realizar metástasis son más altas, según señalan los mismos autores mencionados anteriormente.

Según presencia o ausencia de metástasis, existieron diferencias significativas respecto a la sobrevida, demostrando que aquellos pacientes que poseían metástasis al momento del diagnóstico vivieron menos tiempo, con una sobrevida de sólo 35\% a los cinco años. Vieira et al. (2012) menciona que, si el diagnóstico se realiza en etapas tempranas las tasas de curación van de $80 \%$ a $90 \%$ y la mortalidad presenta $10 \%$ a $15 \%$ de ocurrencia. Por otra parte, cuando ya existe metástasis es porque el diagnóstico fue tardío, en cuyo caso el promedio de sobrevida a los cinco años cae a $25 \%$. Esto se debe principalmente a que si el carcinoma presenta metástasis es difícil su erradicación completa, ya que continuamente aparecerán nuevos focos en diferentes partes del cuerpo ${ }^{8}$.

El tratamiento más frecuente fue cirugía resectiva (40\% del total de pacientes tratados) tal como en el estudio de Gutiérrez-Pascual et al. (2011), donde fue $91 \%$ de los casos. Ésta es la modalidad de tratamiento más realizada en los pacientes con carcinoma de labio, donde se hace una resección en forma de cuña con una recidiva de sólo $8 \%$, presentando una sobrevida a los cinco años de $95 \%$ a $100 \%{ }^{8}$. Radioterapia fue el tratamiento con peor sobrevida debido a que cuando se realizaron tratamientos paliativos, dado que los tumores eran inoperables, éste fue el procedimiento más utilizado (5 de 7 casos).

En nuestro estudio la sobrevida a los cinco años para el CEC de labio en Chile fue aproximadamente $73 \%$, bastante diferente a los Estados Unidos de Norteamérica que, según cifras SEER, posee una sobrevida a los cinco años de $89,5 \%$; sin embargo, nuestros resultados no distan considerablemente de los resultados de otros estudios en los que la sobrevida fue $78 \%$ a $79 \%$ a los cinco años $^{3,4}$. Esta diferencia con Estados Unidos de Norteamérica puede explicarse por la falta de conocimientos por parte de la población sobre este tipo de cáncer; también hay falta conocimientos de los profesionales de la salud debido a que muchas veces se desconoce su presentación clínica y, aun siendo una patología de fácil detección, no es detectada a tiempo. Además, existe falta de cuidado por parte de la población respecto a la radiación solar y los daños que ésta puede causar. Es necesario crear programas de salud que aporten mayor información a la población y fomenten el autocuidado. El carcinoma labial es una patología de fácil diagnóstico y normalmente es visible, a diferencia de otros cánceres del cuerpo que, si bien son igualmente silenciosos, no son visibles. Si el carcinoma es detectado en forma temprana llega a tener una sobrevida de $90 \%$ en ausencia de metástasis, por esta razón es necesario crear conciencia en la población y así evitar fallecimientos por esta causa.

Agradecimientos: Al Instituto Nacional del Cáncer y a Mario Ortiz por su constante apoyo durante la etapa de realización de esta investigación.

\section{Referencias}

1. Bilkay U, Kerem H, Ozek C, Gundogan H, Guner U, Gurler T, et al. Management of lower lip cancer: a retrospective analysis of 118 patients and review of the literature. Ann Plast Surg 2003; 50 (1): 43-50.

2. Busick TL, Uchida T, Wagner RF. Preventing ultraviolet light lip injury: beachgoer awareness about lip cancer risk factors and lip protection behavior. Dermatol Surg 2005; 31 (2): 173-6.

3. Czerninski R, Zini A, Sgan-Cohen HD. Lip cancer: incidence, trends, histology and survival: 1970-2006. Br J Dermatol 2010; 162 (5): 1103-9.

4. de Visscher JG, van den Elsaker K, Grond AJ, van der Wal JE, van der Waal I. Surgical treatment of squamous cell carcinoma of the lower lip: evaluation of long-term 
results and prognostic factors-a retrospective analysis of 184 patients. J Oral Maxillofac Surg 1998; 56 (7): 81420; discussion 20-1.

5. Gutiérrez-Pascual M, Vicente-Martín FJ, Fernández-Álvarez JG, Martín-López R, Pinedo-Moraleda F, López-Estebaranz JL. Squamous cell carcinoma of the lip. A retrospective study of 146 patients. J Eur Acad Dermatol Venereol 2012; 26 (9): 1116-21.

6. Jayaprakash V, Reid M, Hatton E, Merzianu M, Rigual $\mathrm{N}$, Marshall J, et al. Human papillomavirus types 16 and 18 in epithelial dysplasia of oral cavity and oropharynx: a meta-analysis, 1985-2010. Oral Oncol 2011; 47 (11): 1048-54.

7. Moore S, Johnson N, Pierce A, Wilson D. The epidemiology of lip cancer: a review of global incidence and aetiology. Oral Dis 1999; 5 (3): 185-95.

8. Neville B. Epithelial Pathology. En: Neville B, Damm D, Allen C, Bouquot J, Dolan John, Oral and Maxillofacial Pathology. St. Louis, Estados Unidos: Editorial Saunders-Elsevier, 2009. p. 409-15.

9. Pogoda JM, Preston-Martin S. Solar radiation, lip protection, and lip cancer risk in Los Angeles County women (California, United States). Cancer Causes Control 1996; 7 (4): 458-63.

10. Prelec J, Laronde D. Treatment modalities of oral cancer. Canadian Journal of Dental Hygiene 2014; 48 (1): 13-9.

11. Pukkala E, Söderholm AL, Lindqvist C. Cancers of the lip and oropharynx in different social and occupational groups in Finland. Eur J Cancer B Oral Oncol 1994; 30B (3): 209-15.

12. Radoi L, Luce D. A review of risk factors for oral cavity cancer: the importance of a standardized case definition. Community Dent Oral Epidemiol 2013; 41 (2): 97-109, e78-91.

13. Salgarelli AC, Sartorelli F, Cangiano A, Pagani R, Collini M. Surgical treatment of lip cancer: our experience with 106 cases. J Oral Maxillofac Surg 2009; 67 (4): 840-5.

14. Veness MJ, Ong C, Cakir B, Morgan G. Squamous cell carcinoma of the lip. Patterns of relapse and outcome: Reporting the Westmead Hospital experience, 19801997. Australas Radiol 2001; 45 (2): 195-9.

15. Vieira RA, Minicucci EM, Marques ME, Marques SA. Actinic cheilitis and squamous cell carcinoma of the lip: clinical, histopathological and immunogenetic aspects. An Bras Dermatol 2012; 87 (1): 105-14.

16. Viviano M, Addamo A, Lorenzini G. Oral Cancer. Int J Clin Dent 2013; 6 (3): 291-311.

17. Vukadinovic M, Jezdic Z, Petrovic M, Medenica LM, Lens M. Surgical management of squamous cell carcinoma of the lip: analysis of a 10-year experience in 223 patients. J Oral Maxillofac Surg 2007; 65 (4): 675-9.

18. Warnakulasuriya S. Global epidemiology of oral and oropharyngeal cancer. Oral Oncol 2009; 45 (4-5): 309-16.

19. World Health Organization (WHO). GLOBOCAN, International Agency for Research on Cancer (IARC). 2014. (Disponible en http://globocan.iarc.fr).

20. Yako-Suketomo H, Marugame T. Comparison of time trends in lip cancer incidence (1973-97) in East Asia, Europe and USA, from Cancer Incidence in Five Continents, Vols IV-VIII. Jpn J Clin Oncol 2008; 38 (6): 456-7.

21. Zitsch RP. Carcinoma of the lip. Otolaryngol Clin North Am 1993; 26 (2): 265-77.23. 